\title{
Oxidation shuts down an auto-inhibitory mechanism of von
}

\author{
Willebrand factor \\ Rachel Tsai and Gianluca Interlandi* \\ Department of Bioengineering, University of Washington, 98195 Seattle, WA, USA \\ *Correspondence to: gianluca@u.washington.edu
}

\begin{abstract}
Recently, the platelet-binding function of the blood protein von Willebrand factor (VWF) has been shown to be activated by oxidizing conditions such as created by inflammation. This observation has been linked to the oxidation of methionine residues in three tandem A domains of VWF. Here, we used a dynamic flow assay to investigate which auto-inhibitory mechanisms of VWF are shut down leading to the observed activation. The results show that oxidizing agents do not directly activate the A1 domain, which is the domain in VWF that contains the binding site to platelet surface receptors, but are likely to remove the inhibitory function of neighboring domains of A1.
\end{abstract}

\section{Introduction}

von Willebrand Factor (VWF) is a multimeric plasma protein whose primary function is to recruit platelets to the site of blood vessel injury. This is mediated by the VWF A1 domain binding to platelet surface receptor glycoprotein $\mathrm{Ib} \alpha(\mathrm{GpIb} \alpha)[1]$. During the inflammatory response, neutrophils are activated and release hydrogen peroxide (H2O2), which is converted to hypochlorous acid ( $\mathrm{HOCl})$ through the action of myeloperoxidase (MPO). It is known that $\mathrm{HOCl}$ oxidizes methionine residues in blood proteins. Recently, it has been shown that oxidation 
increases binding of VWF to platelets [2], but the molecular mechanism is not well understood.

Our goal is to study whether the A2 and A3 domains, which are located C-terminally to A1, play a role in the oxidant-induced activation of VWF. These domains are critical in how VWF tethers to platelets. The A2 domain contains a site that is cleaved by the metalloprotease ADAMTS13 converting VWF multimers to smaller and less active forms [1]. The A3 domain attaches to exposed collagen on the site of vascular damage [1]. A critical question is whether oxidation causes activation in only the A1 domain or if it decreases the inhibitory effect of the neighboring A1 and A2 domains. Understanding how oxidation alters the function of VWF is necessary to explain the increased risk of thrombosis in patients suffering of chronic or acute inflammation [1]. Here, we use a dynamic flow assay to study how oxidation affects the adhesion of GpI $\alpha-$ coated beads to constructs of VWF consisting of either the isolated A1 domain or the A1, A2 and A3 domains covalently linked to each other.

\section{Methods}

First, plasmids containing biotin-tagged constructs of VWF with different combinations of domains are transfected into Chinese hamster ovary (CHO) cells with lipofectamine 2000 (Invitrogen, Carlsbad, CA) for five hours at $37^{\circ} \mathrm{C}$. Two constructs have been expressed for the dynamic flow assay: "longA1" contains the A1 domain including a 34-amino acids N-terminal linker, and "longA1A2A3" containing the A1, A2 and A3 domains and a 34-amino acids Nterminal linker. The $\mathrm{CHO}$ cells are incubated in $12.5 \mathrm{~mL}$ of DMEM culture medium (Invitrogen, Carlsbad, CA) that contains 10\% tetracycline-free Fetal Bovine Serum (Atlanta Biologicals, Lawrenceville GA) and biotin for 72 hours at $37^{\circ} \mathrm{C}$. Then media for these constructs are collected and concentrated using Amicon Ultra-15 Centrifugal Filters (EMD Millipore, Billerica 
MA). The centrifugation tubes are size exclusive, preventing large molecules like our construct from passing through the filter and as a result concentrate our construct $5 \mathrm{X}$ from $12.5 \mathrm{~mL}$ to 2.5mL. After concentration, the media is eluted through a desalting column, PD-10 (GE Healthcare, Marlborough, MA) to remove excess biotin. This removal is necessary because we need biotin to attach to streptavidin sites for the flow chamber experiments, identification and quantification. Having excess biotin would cause non-specific binding and low adhesion of beads to the plate in flow chamber experiments. A western blot is then performed to detect and identify the protein using a streptavidin-HRP antibody (SA-HRP AB). The streptavidin portion of the antibody will bind to the exposed biotin on our VWF construct. The HRP portion (horseradish peroxidase) will be used with a chemiluminescent SuperSignal detection kit (Thermo Fisher Scientific Waltham, MA). The SA-HRP AB will attach to VWF constructs that are on a nitrocellulose paper. The HRP portion in conjugation with the SuperSignal kit will cause luminescence in $425 \mathrm{~nm}$ range, which is exposed onto an x-ray film and developed. A protein ladder helps to identify the size and presence of the VWF constructs.

Next, 35-mm tissue culture polystyrene dishes were coated with biotinylated bovine serum albumin (biotin-BSA), incubated with streptavidin at $37^{\circ} \mathrm{C}$ and finally incubated with a specific VWF construct. Because streptavidin contains four biotin binding sites, it binds to both biotin-BSA and the constructs forming a protein sandwich. Beads coated with GPIb $\alpha$ are similarly made by expressing GC300-b, which is the N-terminal 300 residues of GPIb $\alpha$ with a Cterminal biotin tag to mimic their orientation on platelets.

Oxidation of the VWF constructs was achieved by incubating the plates (with the constructs already anchored as described above) with $5 \mu \mathrm{M} \mathrm{HOCl}$. We estimated that these concentrations will yield molar ratios of 1:50 of VWF protein molecules to oxidant, which is of 
similar magnitude as the molar ratios present in a previous oxidation study [2]. The oxidation reaction will be quenched by adding free methionine.

The prepared plates were inserted into a Glycotech ${ }^{\mathrm{TM}}$ flow chamber connected to a syringe pump (Figure 1). GpIb $\alpha$-coated beads were injected into the flow chamber and allowed to settle. A CCD video camera recorded the behavior while flow was controlled to produce $1.4 \mathrm{dyne} / \mathrm{cm}^{2}$ of shear stress, increased stepwise to $30 \mathrm{dyn} / \mathrm{cm}^{2}$, then decreased again. The beads were tracked and number adherent and average velocity measured using the ImageJ and SVCell® software packages (Figure 2). Because of the adhesive properties of the A1 domain binding to GpIb $\alpha$, beads adhered and rolled on the surface coated with VWF constructs. Velocities and ratios of adhering beads were compared between oxidized and non-oxidized constructs. Using a biotin-4fluorescein (b4f) assay, and it was found that adding oxidant does not alter the surface concentration of VWF protein.

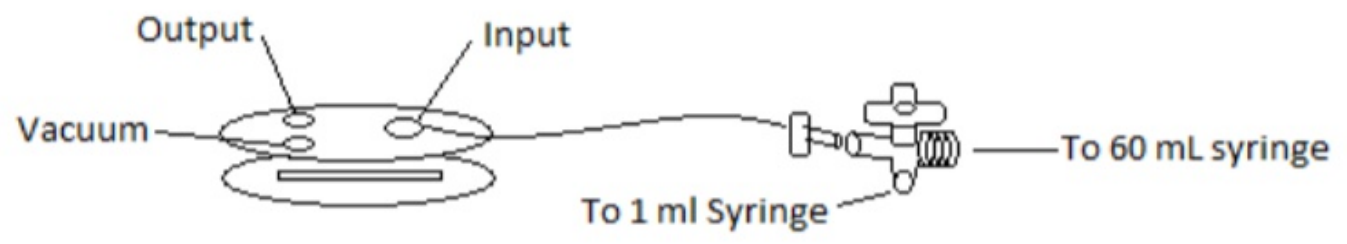

Figure 1: Illustration of a dynamic flow assay using a flow chamber. 


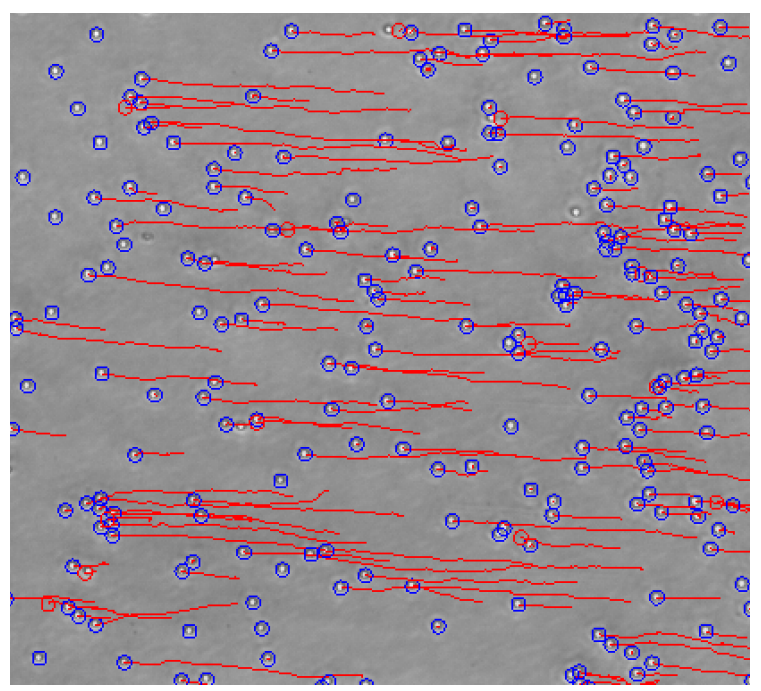

Figure 2: Tracking Bead Movement with SVCell@.

\section{Results}

The following VWF constructs were expressed and tested in the dynamic flow assay: isolated A1 domain and the A1-A2-A3 complex. Both constructs contained the entire A1 N-terminal linker. The surface was washed with GpIb $\alpha$-coated beads and oxidized versus non-oxidized VWF constructs were compared. In the case of isolated A1, oxidation caused a slight reduction in rolling velocities of beads rolled slightly at higher shear, although the difference is not statistically significant except at high shear (Figure 3a). Furthermore, there is no statistically significant difference in the ratio of bound beads for isolated A1 whether this is oxidized or not (Figure 3a). In contrast, when A1A2A3 was attached to the surface, oxidation caused a statistically significant lower rolling velocities at both intermediate and high shear compared to the situation where non-oxidized A1A2A3 was used (Figure 3b). Also, a statistically significant larger ratio of bound beads was observed at high shear (and marginally statistically significant at intermediate shear) when A1A2A3 was oxidized compared to when it was not oxidized (Figure $3 b)$. It is important to note that the slight decrease in rolling velocities observed with the oxidized 
A1 domain could be attributed to an initial lower surface coverage (Figure 3c) causing weakly bound beads to detach early and leading to an artificially lower average rolling velocity.
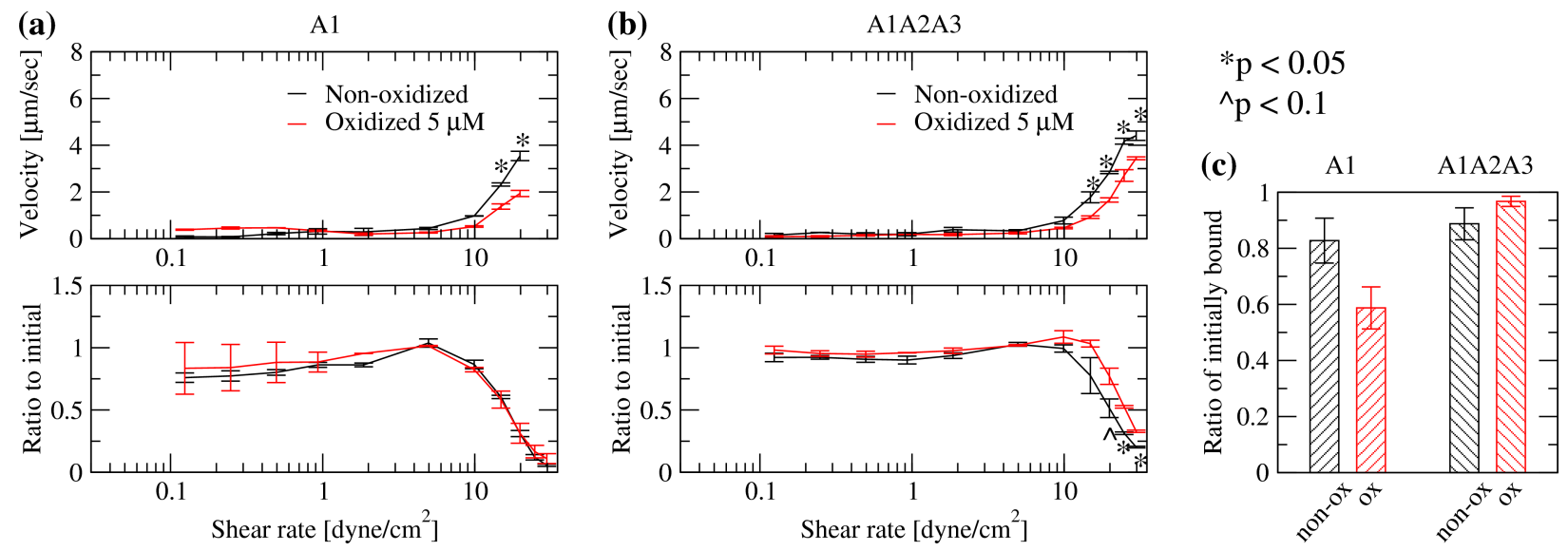

Figure 3: Results from the dynamic flow assay. Rolling velocities and ratio of bound to initially bound beads for (a) isolated A1 domain containing the N-terminal linker, (b) VWF A1-A2-A3 construct. (c) Ratio of initially bound (after turning shear on) to initially present beads (before). Reported are averages and standard errors of the mean for two measurements. p-values were calculated from a Student's t-test. A difference with a p-value smaller than 0.05 or smaller than 0.1 was considered to be statistically or marginally statistically significant, respectively.

\section{Discussion}

A previous study showed that oxidation enhances the platelet-binding function of VWF and linked this observation to the conversion of methionine residues in the A1, A2 and A3 domains to methionine sulfoxide [2]. Here, we investigated whether oxidation directly activates the A1 domain, which contains the binding site to platelet surface receptor GpIb $\alpha$, or whether oxidation removes the inhibitory function of neighboring domains. The results show that oxidation is not likely to directly affect the A1 domain itself but to rather diminish the inhibitory action that the A2 and A3 domains have on the A1 domain (Figure 3). Support for this model is provided by 
previous evidence that the A2 and A3 domains inhibit A1 binding to GpIba [3,4]. Furthermore, a previous molecular dynamics study by us indicated that methionine oxidation leads to a decrease in the tensile force required to unfold the A2 domain and to the thermodynamic destabilization of its fold [5]. It is important to note that ADAMTS13, which normally cleaves the A2 domain under non-oxidizing conditions converting VWF multimers to smaller and less active forms, is no longer able to cut the A2 domain once this is oxidized [6]. Thus, it is plausible that oxidant removes the inhibitory function of the A2A3 complex in the flow chamber experiments by causing the A2 domain to unfold and no longer obstruct the binding site in the A1 domain to GpIb $\alpha$. In addition to destabilizing the A2 domain fold, it is also possible that oxidation directly impairs binding between A1 and the A2A3 complex by oxidizing methionine residues located at the inter-domain interfaces.

Both, the A1 and A1A2A3 constructs were expressed with a 34-amino acids long Nterminal linker. This has been previously shown to also have an inhibitory effect on A1 [7]. The experiments performed here indicate that oxidation may slightly reduce this inhibitory effect, although the resulting activation was much less significant than in the case where the A1A2A3 construct was tested (Figure 3). Furthermore, it is also possible that, in addition to removing the inhibitory function of the A2A3 domains complex, methionine oxidation also disrupts the interaction between A1 and the N-terminally located D'D3 domains, which have also been shown to inhibit A1 binding to GpIb $\alpha[8,9]$. In conclusion, this study suggests that oxidation is likely to shut down at least one of the auto-inhibitory mechanisms in VWF consisting in the inhibition exerted by the $\mathrm{A} 2$ and $\mathrm{A} 3$ domains on the $\mathrm{A} 1$ domain. 


\section{Acknowledgements}

This research was made possible thanks to a career training award from the National Institutes of Health K25HL118137 to GI.

\section{References}

[1] Yip et al, "Primary Platelet Adhesion Receptors", IUBMB Life, 57: 103-108, 2005

[2] Fu X, Chen J, Gallagher R, Zheng Y, Chung DW, López JA. Shear stress-induced unfolding of VWF accelerates oxidation of key methionine residues in the A1A2A3 region. Blood. 2011;118(19):5283-5291. doi:10.1182/blood-2011-01-331074.

[3] Auton M, Sowa K, Smith S, Sedlak E, Vijayan V, Cruz MA. Destabilization of the A1

Domain in von Willebrand Factor Dissociates the A1A2A3 Tri-domain and Provokes Spontaeous Binding to Glycoprotein Ib $\alpha$ and Platelet Activation under Shear Stress. J. Biol. Chem. 285:22831.

[4] Martin C, Morales LD, Cruz MA. Purified A2 domain of von Willebrand factor binds to the active conformation of von Willebrand factor and blocks the interaction with platelet glycoprotein Iba. J. Thromb. Haemost. 5:1363-1370, 2007.

[5] Interlandi G. Destabilization of the von Willebrand factor $\{A\} 2$ domain under oxidizing conditions investigated by molecular dynamics simulations. PLOS ONE. 13(9):e0203675, 2018 [6] Chen J, Fu X, Wang Y, Ling M, McMullen B, Kulman J, Chung DW, Lopez JA. Oxidative modification of von Willebrand factor by neutrophil oxidants inhibits its cleavage by ADAMTS13. Blood. 115(3):706-712, 2010 
[7] Interlandi G, Yakovenko O, Tu AY, Harris J, Le J, Chen J, Lopez JA, Thomas WE. Specific electrostatic interactions between charged amino acid residues regulate binding of von Willebrand factor to blood platelets. J. Biol. Chem. 292(45):18608-18617, 2017

[8] Ulrichts H, Udvardy MS, Lenting PJ, Pareyn I, Vandeputte N, Vanhoorelbeke K, Deckmy H. Shielding of the A1 domain by the D'D3 domains of von Willebrand factor modulates its interaction with platelet glycoprotein Ib-IX-V. J. Biol. Chem. 281(8):4699--4707, 2006

[9] Madabhushi SR, Zhang C, Kelkar A, Dayananda KM, Neelamegham S. Platelet Gplb alpha Binding to von Willebrand Factor Under Fluid Shear: Contributions of the D'D3-Domain, A1Domain Flanking Peptide and O-Linked Glycans. J. Am. Heart. Assoc. 3(5), 2014 